\title{
Assessment of orofacial pain management in a pediatric emergency department and at home after discharge
}

\author{
Yurena Aguilar de la Red, B.S. ${ }^{a}$, Gema Manrique Martín, B.S. ${ }^{a}$, \\ Gloria Guerrero Márquez, Nursea, Concepción González Herrero, Nursea, \\ Paula Vázquez López, M.D. ${ }^{a}$ and Concepción Míguez Navarro, M.D. ${ }^{a}$
}

\begin{abstract}
Introduction. An inadequate pain management is common in the emergency department. Our objective was to analyze pain management among children with an orofacial infection or trauma in the emergency department and to assess compliance and satisfaction with analgesia prescribed at discharge.

Population and methods. Cross-sectional, observational and analytical study in children attending the emergency department for an orofacial infection or trauma over 2 months. Pain management in the emergency department, analgesia prescribed at home and, following a call to parents, treatment provided and its adequacy to control pain were registered.

Results. In total, 252 patients (mean age: 4.5 years, SD: 3.89) were included. Pain assessment was recorded at the triage for $8.7 \%$, and in the medical report, for $3.6 \%$. Analgesia was administered to $41.3 \%$ in the emergency room. At discharge, no analgesia was prescribed to $13.9 \%$; scheduled analgesia, to $25.4 \%$; and as needed, to $60.3 \%$. Pediatricians prescribed scheduled analgesia more frequently than surgeons $(34.4 \%$ versus $16.5 \%, p<0.01$ ).

At home, no analgesia was administered to $39.3 \%$; scheduled analgesia, to $36.1 \%$; and as needed, to $23 \%$. There is little correlation between prescription at discharge and at home (Kappa: 0.178 ). Analgesia was considered adequate in $84 \%$ of cases, and was more frequent in trauma injuries than in infections ( $85.7 \%$ versus $14.3 \%$, $p<0.01$ ).

Conclusions. Pain assessment and management was scarce in the emergency department. The most common prescription was as needed, contrary to what is recommended in the guidelines. Analgesic control worked better for
\end{abstract} trauma injuries than for infections.

Key words: analgesia, emergency department, orofacial, pediatrics.

http:/ / dx.doi.org/ 10.5546/ aap.2018.eng.28

To cite: Aguilar de la Red Y, Manrique Martín G, Guerrero Márquez G, et al. Assessment of orofacial pain management in a pediatric emergency department and at home after discharge. Arch Argent Pediatr 2018;116(1):28-34.

\section{INTRODUCTION}

The International Association for the Study of Pain (IASP) defines pain as "an unpleasant sensory and emotional experience associated with actual or potential tissue damage, or described in terms of such damage". ${ }^{1}$

Although pain is a common symptom seen at the emergency department, ${ }^{2}$ several studies have demonstrated that pain management is inadequate. ${ }^{3}$ There are multiple causes for this: difficulty to assess pain, fear to use strong analgesics, and use of medications on an as needed basis instead of at scheduled intervals. The implementation of protocols improves the outcomes of pain management. ${ }^{4,5}$

Health care providers from the emergency department should recognize and treat pain promptly. ${ }^{6}$ To this end, first it is necessary to identify and assess pain intensity using scales based on age and cognitive level.? Then, a drug should be selected according to the type of pain and its intensity, side effects, concomitant conditions, and administration route. Effective analgesics should be prescribed on a scheduled basis instead of as needed, during the first 48-72 hours, and the possibility of using rescue analgesics ${ }^{8}$ or other associated strategies, such as applying cold or heat, ${ }^{9}$ should be taken into consideration.

Pain management should be included as part of patient care because it affects not only patient satisfaction and well-being but also the outcomes of the underlying condition. ${ }^{7}$ The orofacial region has an extensive nociceptive innervation, and any injury will invariably cause 
pain. No studies have been found regarding the assessment and management of pain in the oral and facial regions in the pediatric emergency department (PED). For this reason, a study was designed with the primary objective of describing pain management in children with infectious or traumatic orofacial pathologies since the moment they arrive at the PED and the subsequent management at home. The secondary objective was to assess parental opinions regarding the effectiveness of prescribed analgesia.

\section{POPULATION AND METHODS}

\section{Subjects and study location}

The study was conducted at the Pediatric Emergency Department (PED) of Hospital Universitario Gregorio Marañón, a tertiary care facility located in Madrid (Spain), where approximately 55000 pediatric emergencies are seen annually.

The medical records of patients seen in the emergency department were reviewed, and children younger than 16 years who attended the PED for an infectious or traumatic orofacial pathology between October and November 2015 were included. Parents/tutors who refused their consent, those assessed by any of the investigators (the rest of the health care team and nurse staff were blinded to the study), and those whose language was a barrier for the interview were excluded.

Patients were assessed by physicians and surgeons based on the PED's triage criteria.

\section{Design}

A cross-sectional, observational, and analytical study was designed with the subjects and in the setting described above.

\section{Study protocol}

Candidates were identified based on the medical records of our PED. Between 7 and 10 days after the visit to the PED, investigator physicians contacted parents / tutors on the phone, described the study, and asked for their verbal informed consent to participate. A maximum of 5 calls were made, in no case exceeding the 10day period since the first visit to the PED. For patients whose parents/tutors had given the consent, a brief survey was completed based on a pre-established script that lasted approximately 5 minutes. The survey included objective data on the administered analgesia and subjective data on treatment response and satisfaction (Table 1). Then data were collected retrospectively from the medical record written during the visit (Table 2).

\section{Definitions}

Type of analgesia

- Scheduled: administered at regular intervals, regardless of the presence of pain.

- As needed: administered based on the presence of pain.

Type of health care provider and his/her area

- Pediatrician: in our study, a pediatrician assessed infections, trauma injuries, and wounds that could not be repaired.

TABLE 1. Clinical outcome measures and interview with parents

\begin{tabular}{|c|c|}
\hline Clinical (primary) outcome measures & Interview with parents (secondary outcome measures) \\
\hline $\begin{array}{l}\text { - Mean age } \\
\text { - Sex } \\
\text { Diagnosis: } \\
\text { - Oral trauma } \\
\text { - Facial trauma } \\
\text { - Oral infection } \\
\text { - Facial infection } \\
\text { - Oral wound } \\
\text { - Facial wound } \\
\text { Pain assessment recorded in the triage report and } \\
\text { the medical record (yes/no) } \\
\text { - Analgesia administered in the emergency room } \\
\text { Analgesia prescribed to be taken at home (type/drug): } \\
\text { - No analgesia/scheduled analgesia/on an as-needed } \\
\text { - basis/rescue } \\
\text { Adjunct therapy at home: } \\
\text { - Yes/no } \\
\text { - Which one? }\end{array}$ & $\begin{array}{l}\text { 1. Did you administer analgesia to your child at home? Yes/no. } \\
\text { What was the route of administration? Oral/topical. } \\
\text { How often did you administer it? } \\
\text { Was it at scheduled intervals or as needed? } \\
\text { 2. What do you think of the effectiveness of the scheduled } \\
\text { pain treatment? } \\
\text { Good/regular/poor. } \\
\text { 3. Do you think the pain treatment regimen was adequate? } \\
\text { Adequate / regular/inadequate. } \\
\text { 4. Has your child's intake reduced? } \\
\text { No/partially/ a lot. } \\
\text { 5. Did you have to seek care in other center due to pain? } \\
\text { Yes/no. } \\
\text { What type of center? } \\
\text { 6. What is your level of satisfaction with analgesia? } \\
\text { 0-10 scale (0: not at all satisfied; } 10 \text { : completely satisfied). }\end{array}$ \\
\hline
\end{tabular}


- Surgical specialist: pediatric and maxillofacial surgeons repairing wounds in the oral and facial regions, respectively.

Diagnoses

To facilitate result interpretation, the following categories were encompassed and subsequently subdivided based on location (facial or oral region).

- Wound: an injury with loss of continuity.

- Trauma: an injury with no loss of continuity.

- Infection: aphthous stomatitis, cellulitis, parulides, etc.

Adequacy of prescribed treatment

Parental opinion regarding the adequacy of the treatment prescribed for their children's pain. It was defined as follows:

- Adequate: pain is appropriately controlled with compliance of the prescribed treatment.

- Inadequate: the child had pain in spite of compliance.

- Regular: an intermediate situation between an adequate and an inadequate treatment.

Level of satisfaction:

Feeling satisfied with prescribed analgesia in a 0-10 scale, where 0 meant not at all satisfied and 10 , very satisfied.

\section{Ethical considerations}

The study was approved by the local ethics and clinical research committee, was in accordance with the Declaration of Helsinki standards and with full respect for the good clinical practice principles. It was classified by the Spanish Agency of Medicinal Products and Medical Devices (Agencia Española del Medicamento y Productos Sanitarios) as a "prospective follow-up post-authorization study (EPA-SP)." The verbal informed consent was obtained from all parents/tutors of included children.

\section{Statistical analysis}

Quantitative outcome measures were reported as mean and standard deviation, while qualitative outcome measures were described as frequency and percentage. The $\chi^{2}$ test or a the MannWhitney $\mathrm{U}$ test were used to compare qualitative or quantitative outcome measures, respectively, and the Kappa's correlation coefficient was used to study agreement among categorical outcome measures. The SPSS software (version 20.0.1) was used for analysis, and a $p$ value $<0.05$ was considered statistically significant.

\section{RESULTS}

\section{Patient selection and characteristics}

Over the 2-month study period, 10171 children were seen at the PED; of them, 302 $(2.97 \%)$ had an infectious or traumatic orofacial pathology. A total of 252 patients were included (Figure 1). The characteristics of patients are described in Table 2.

\section{Results}

The pain assessment was recorded for 29 patients (11.5\%). In 22 cases, the pain assessment was included in the triage report $(8.7 \%)$ and in 9 cases, in the medical report $(3.6 \%)$ (7 trauma injuries/wounds and 2 infections). Two patients had a pain assessment included in the triage and medical reports, and a validated scale was used to $1(0.4 \%)$.

Analgesia was given in the emergency room to 104 patients $(41.3 \%)$ : topical or local analgesia was administered to $93(89.4 \%)$ and oral systemic analgesia, to $11(10.6 \%)$ (acetaminophen: $36.3 \%$; ibuprofen: $36.3 \%$; dipyrone: $27.4 \%$ ).

At the time of discharge, analgesia was prescribed to 217 patients $(86.1 \%)$, whereas 153 patients received analgesia at home $(60.7 \%)$. The

TABLE 2. Sample characteristics, $N=252$

\begin{tabular}{lcccc}
\hline & Total & Facial region & Oral cavity & Significance $(p)$ \\
\hline $\begin{array}{l}\text { Mean age in years (SD) } \\
\text { Sex (\% of boys) }\end{array}$ & $4.5(3.9)$ & $4.64(3.9)$ & $4.43(3.89)$ & 0.49 \\
Observer physician & 57.9 & 61.7 & 49.4 & 0.06 \\
$\quad$ Pediatrician & $49.6 \%(125)$ & $37.7 \%(66)$ & $76.6 \%(59)$ & $<0.01$ \\
$\quad$ Surgeon & $50.4 \%(127)$ & $62.3 \%(109)$ & $23.4 \%(18)$ & \\
$\quad$ Diagnosis at the time of discharge & & & \\
$\quad$ Wound & $44.8 \%(113)$ & $55.4 \%(97)$ & $20.8 \%(16)$ & $<0.01$ \\
$\quad$ Trauma & $34.9 \%(88)$ & $38.9 \%(68)$ & $26 \%(20)$ & \\
$\quad$ Infection & $20.3 \%(51)$ & $5.7 \%(10)$ & $53.2 \%(41)$ & \\
\hline
\end{tabular}

SD: standard deviation. 
type of regimen is described in Figure 2. Poor agreement was observed between analgesia prescribed at the time of discharge and that administered at home (Kappa: 0.178).

Pediatricians prescribed analgesia at the time of discharge to $80.8 \%$ of patients (101 out of 125 patients seen) whereas surgeons, to
91.3\% (116 out of 127 patients), although the former prescribed scheduled analgesia more frequently than the latter (43: $34.4 \%$ and $21: 16.5 \%$, respectively, $p<0.01$ ).

The most common drug prescribed in a scheduled manner was ibuprofen, to 195 patients $(90.3 \%)$, followed by acetaminophen, prescribed

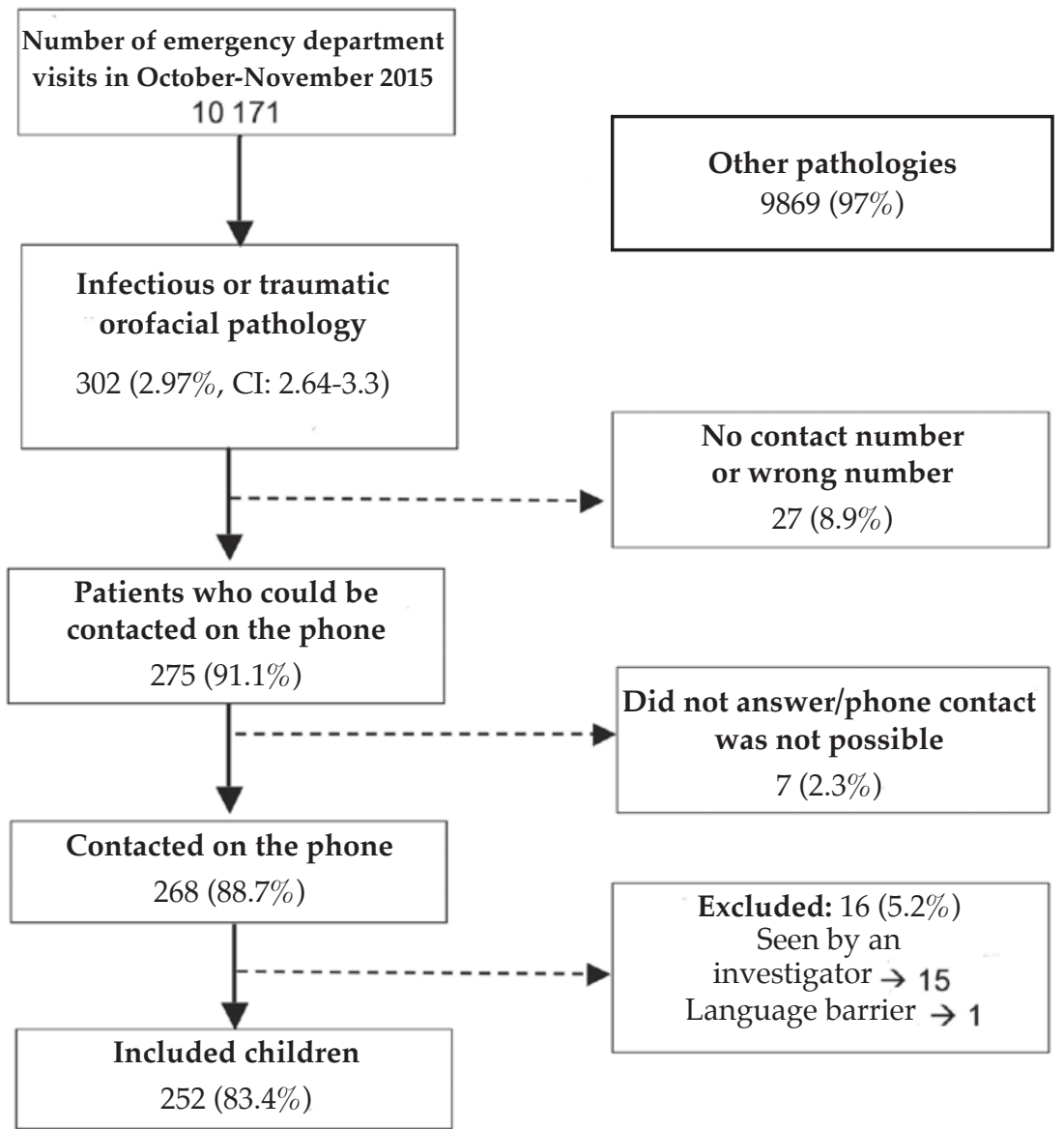

FIGURE 2. Type of analgesia prescribed at the time of discharge and administered at home. $N=252$

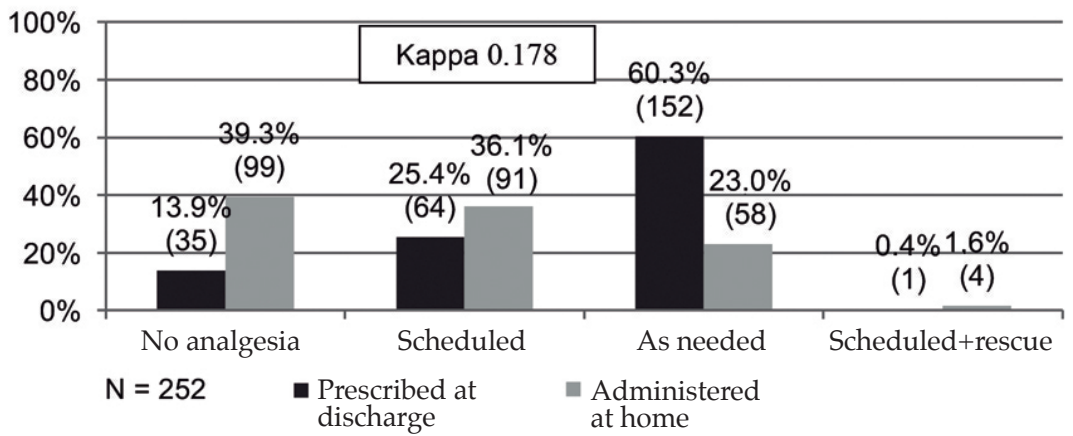

Kappa's coefficient statistical test. 
to $18(8.3 \%)$. Topical oral anesthesia was indicated to 31 patients $(12.3 \%)$ and cryotherapy, to 48 $(19.1 \%)$. At home, cryotherapy was administered as adjunct therapy to 54 patients $(22 \%)$ and topical analgesia, to $37(22 \%)$.

Parents subjectively referred a reduction in their children's intake (expressed as a partial or major reduction) in 44 cases $(17.4 \%)$; of these, $39(88.6 \%)$ had an oral pathology and 5 , a facial one $(11.4 \%), p<0.001$. Eleven patients $(4.4 \%)$ had to return for a reconsultation. The proportion of reconsultations was higher among children with a reduced intake $(18.2 \%)$ than among those who had maintained their normal intake $(1.4 \%)(p<0.01, \mathrm{OR}=15.19,95 \%$ confidence interval [CI]: 3.85-54.96). No differences were observed once reconsultations were analyzed based on injury location $(p=0.349)$. To avoid any potential interference of location on intake, a regression model was done maintaining similar significance levels $(p<0.01$ for intake and $p=0.366$ for location).

Parents considered that prescribed analgesia was adequate for their children's level of pain in 212 cases $(84.1 \%)$, regular in $37(14.7 \%)$, and inadequate in $3(1.2 \%)$. It was more commonly considered adequate among patients with trauma injuries and wounds than among those with an infectious pathology (Figure 3). In addition, in the first case, analgesia was prescribed at the time of discharge more frequently than in the second case $(91.7 \%$ versus $74.2 \%)$. The level of satisfaction with prescribed analgesia was $9.17( \pm 1.2)$ among patients with trauma injuries, $9.24( \pm 1.12)$ among those with wounds, and $8.86( \pm 1.46)$ among those with an infectious pathology, $p=0.41$.

\section{DISCUSSION}

Pain is a highly prevalent symptom seen in PEDs. ${ }^{2}$ In spite of this, oligoanalgesia and inadequate pain management constitute a phenomenon that is still a major problem in clinical practice, ${ }^{5,10,11}$ as previously seen and demonstrated in our setting. The main problems that have been detected are the scarce assessment of pain, the use of treatments as needed instead of at scheduled intervals, and the administration of analgesics in the emergency room to less than a half of patients. No other article has been found that compared treatment at the time of discharge from the PED and that administered by parents at home. In spite of poorly adequate prescriptions at the time of discharge, it is worth noting that parents administered scheduled analgesia more frequently than as prescribed. Parents more commonly considered that prescribed analgesia was adequate in the case of traumatic pathologies than in that of infectious diseases.

Pain assessment is the previous step to pain management. In spite of this, in our study, there was practically no record of pain assessment carried out in the triage room or included in the medical report. A great disparity was observed in the literature review. The study by Kircher et al. found similar results to those of our study and was conducted in patients with musculoskeletal pain $(6 \%),{ }^{12}$ in contrast to the studies by Benini et al., ${ }^{13}$ done in headache patients, and by $\mathrm{C}$. Johnston et al., ${ }^{14}$ in multiple diseases, where pain was assessed in more than a half of patients $(57 \%$ and $71 \%$, respectively). In our study, and contrary to what is recommended in the literature, pain was assessed without using validated scales., ${ }^{7,15}$

FIGURE 3. Adequacy of analgesia to the child's level of pain according to the parents and based on the pathology. N=252

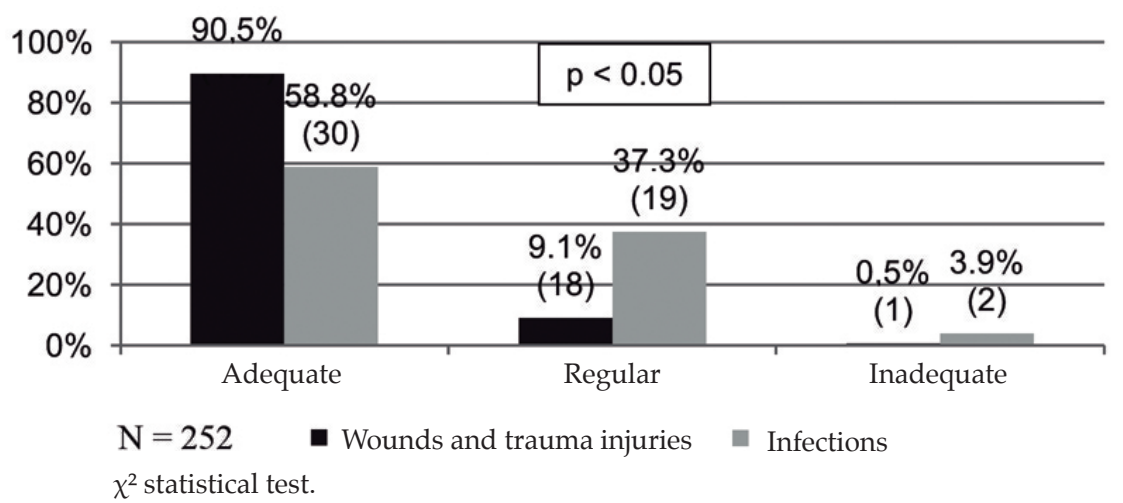


In relation to pain management in the PED, our results were observed to be somewhat better than in other studies ${ }^{12,13,16}$ and similar to those observed by C. Johnston ${ }^{14}$ and $\mathrm{O}^{\prime}$ Donnell, ${ }^{17}$ which included limb trauma cases and where only $39 \%$ and $49 \%$ of patients received analgesia during their stay in the emergency room. In our study, like in the ones by Rogovik ${ }^{16}$ and Benini et al., ${ }^{13}$ analgesia was prescribed at the time of discharge to most patients; whereas, in the studies by C. Johnston et al. ${ }^{14}$ and Kircher et al., ${ }^{12}$ it was only prescribed to $9 \%$ and $24 \%$ of patients, respectively.

It was observed that pediatricians prescribed analgesia less frequently than surgeons. Although no studies comparing this have been found in the literature, in a multicenter study conducted in the United States ${ }^{18}$ in patients with fractures, at the time of discharge, more general practitioners prescribed analgesia than pediatricians. Although this may be related to the type of pathology seen by each specialist, in any case, it reflects the need to improve analgesia prescription among pediatricians. According to parents, analgesic control seemed more adequate in traumatic pathologies than in infectious ones.

In this study, and contrary to what has been recommended in the guidelines, analgesia was prescribed more commonly as needed than at scheduled intervals. However, parents more frequently administered analgesia in a scheduled manner than as prescribed, which may be the result of an inadequate prescription at the time of discharge. Excluding post-operative pain, there is limited literature on how parents manage their children's pain at home, and none of the studies found compared treatment prescribed at the time of discharge from the PED to that given at home. In a study by Zisk et al. that included fracture patients who were prescribed scheduled analgesia at the time of discharge and whose parents were instructed on how to assess children's pain, ${ }^{19}$ more than a half of patients did not receive analgesia or received only one dose, and this differs from the results of this study. In addition, in the study by C. Johnston, ${ }^{14}$ although the authors did not compare prescribed analgesia to that administered by parents, it was observed that, one week later, pain persisted in $5 \%$ of cases (it is worth remembering that analgesia was prescribed to only 9\%). All these data reflect the importance of an adequate pain management by offering precise and concise guidelines to parents.

Even though the proportion of reconsultations due to pain was low, it increased among children with a reduced intake. These data lead to considering that a reduced intake raises concern among parents, either because of the pain or the resulting hyporexia, and that special care should be taken when prescribing analgesia to children whose feeding may be compromised.

The level of satisfaction with analgesia prescribed in the emergency department was high in our study; however, it is worth mentioning that differences were observed in analgesia adequacy among patients with wounds and trauma injuries versus those with infectious pathologies $(90.5 \%$ versus $58.8 \%$ ). Such difference in the subjective assessment made by parents may have multiple causes, such as the fact that it was common that no type of analgesia was prescribed for infectious pathologies compared to the rest of pathologies (25.8\% versus $8.3 \%$ ) or the higher level of resolution of the causing entity (rapid resolution of trauma injuries and wounds, especially those that can be sutured). In addition, the pain expected by parents in trauma injuries and wounds may be closer to reality than in infectious diseases. Therefore, the reasons for considering that analgesia was inadequate are multifactorial, as suggested in different studies, including the one by M. Gill, ${ }^{20}$ where $32 \%$ of parents of children with limb fractures were not satisfied with pain management following discharge from the PED. M. Gill related it to the administration of few analgesia doses, drug adverse reactions, and other factors inherent to the pathology, such as reduced activity, etc.

Most studies published in the literature refer to trauma and post-operative pain but no studies have been found that assessed analgesia administered in the emergency department for orofacial pathologies. Based on the results of our study, improvement measures are necessary both in pain assessment and pain management in the PED and at the time of discharge. Potential interventions could be making a mandatory pain assessment in the triage room and recording it in the medical report, and administering analgesics as early as possible, even in the triage room. The latter would make optimal pain management conditional to early treatment. ${ }^{21,22}$

The following may be considered limitations of this study: first, data were partially collected from medical records and reports, so certain aspects may not have been adequately reflected. However, it was necessary to collect data this way because health care staff seeing patients had to be blinded to the study so as to avoid any 
interference with their routine clinical practice. Prescribed analgesia adequacy and satisfaction with it were extrapolated from a survey administered to parents, so they may have been affected by an inadequate parental assessment of their children's pain. Results are only applicable to our site and may have been different from how other centers work.

\section{CONCLUSIONS}

At our site, a suboptimal management of pain was observed in relation to orofacial trauma and wounds, and pain assessment and treatment in the PED were scarce. Analgesia was most commonly prescribed as needed, unlike what has been recommended in the guidelines. However, at home, scheduled analgesia administration was more frequent than as prescribed. According to parents, analgesic control seemed more adequate in traumatic pathologies than in infectious ones.

\section{REFERENCES}

1. International Association for Study of Pain. IASP Pain Terminology. Seattle, USA. [Accessed on: May 29 $9^{\text {th }}, 2017$ ]. Available at: https: / / www.iasp-pain.org/Taxonomy?na vItemNumber $=576 \#$ Pain

2. Ali S, Chambers A, Johnson DW, et al. Reported practice variationin pediatric painmanagement: a survey of Canadian pediatric emergency physicians. CJEM 2014;16(5):352-60.

3. Zempsky WT, CraveroJP, American Academy of Pediatrics, Committee on Pediatric Emergency Medicine and Section on Anesthesiology and Pain Medicine. Relief of pain and anxiety in pediatric patients in emergency medical systems. Pediatrics 2004;114(5):1348-56.

4. Decosterd I, Hugli $O$, Tamchès E, et al. Oligoanalgesia in the emergency department: short-term beneficial effects of an education program on acute pain. Ann Emerg Med 2007;50(7):462-71.

5. Chang AK, Bijur PE, Holden L, et al. Efficacy of an acute pain titration protocol driven by patient response to a simple query: Do you want more pain medication? Ann Emerg Med 2016;67(5):565-72.

6. Bailey B, Trottier ED. Managing Pediatric Pain in the Emergency Department. Pediatr Drugs 2016;18(4):287-301.

7. Chiaretti A, Pierri F, Valentini P, et al. Current practice and recent advances in pediatric pain management. Eur Rev Med Pharmacol Sci 2013;17(Suppl 1):S112-26.
8. World Health Organization. WHO guidelines on the pharmacological treatment of persisting pain in children with medical illnesses. 2012. [Accessed on: May 29 ${ }^{\text {th }}$, 2017]. Available at: http:/ / apps.who.int/iris/ bitstream/10665/44540/1/9789241548120_Guidelines.pdf.

9. Krauss BS, Calligaris L, Green SM, et al. Current concepts in management of pain in children in the emergency department. Lancet 2016;387(10013):83-92.

10. Stang AS, Hartling L, Fera C, Johnson D, et al. Quality indicators for the assessment and management of pain in the emergency department: A systematic review. Pain Res Manag 2014;19(6):e179-90.

11. Green SM. There is oligo-evidence for oligoanalgesia. Ann Emerg Med 2012;60(2):212-4.

12. Kircher J, Drendel AL, Newton AS, et al. Pediatric musculoskeletal pain in the emergency department: a medical record review of practice variation. CJEM 2014;16(6):449-57.

13. Benini F, Piga S, Zangardi T, et al. Nationwide study of headache pain in Italy shows that pain assessment is still inadequate in paediatric emergency care. Acta Paediatr 2016;105(5):e200-8.

14. Johnston CC, Bournaki M-C, Gagnon AJ, et al. Self-reported pain intensity and associated distress in children aged 4-18 years on admission, discharge, and one-week follow up to emergency department. Pediatr Emerg Care 2005;21(5):342-6.

15. Drendel AL, Brousseau DC, GorelickMH. Pain assessment for pediatric patients in the emergency department. Pediatrics 2006;117(5):1511-8.

16. Rogovik AL, Rostami M, Hussain S, et al. Physician pain reminder as an intervention to enhance analgesia for extremity and clavicle injuries in pediatric emergency. J Pain 2007;8(1):26-32.

17. O'Donnell J, Ferguson L, Beattie T. Use of analgesia in a paediatric accident and emergency department following limb trauma. Eur J Emerg Med 2002;9(1):5-8.

18. Cimpello LB, Khine H, Avner JR. Practice Patterns of Pediatric Versus General Emergency Physicians for Pain Management of Fractures in Pediatric Patients. Pediatr Emerg Care 2004;20(4):228-32.

19. Zisk RY, Grey M, Medoff-Cooper B, et al. The squeaky wheel gets the grease: parental pain management of children treated for bone fractures. Pediatr Emerg Care 2008;24(2):8996.

20. Gill M, Drendel AL, Weisman SJ. Parent Satisfaction with Acute Pediatric Pain Treatment at Home. Clin J Pain 2013;29(1):64-9.

21. Taylor SE, Taylor DM, Jao K, et al. Nurse-initiated analgesia pathway for paediatric patients in the emergency department: A clinical intervention trial. Emerg Med Australas 2013;25(4):316-23.

22. Thomas D, Kircher J, Plint AC, et al. Pediatric pain management in the emergency department: the triage nurses' perspective. J Emerg Nurs 2015;41(5):407-13. 